\title{
Descemet Stripping Automated Endothelial Keratoplasty - Is a Thinner Donor Lamella the Better Choice?
}

\author{
Iva Dekaris*, Maja Pauk, Nataša Drača, Adis Pašalić and Nikica Gabrić \\ University Eye Hospital "Svjetlost", Heinzelova 39, 10000 Zagreb, Croatia
}

\begin{abstract}
Introduction: To evaluate the influence of lamellar thickness on visual recovery after Descemet stripping automated endothelial keratoplasty (DSAEK) and compare the results to penetrating keratoplasty (PK).

Methods: A prospective case series of 20 eyes with pseudophakic bullous keratopathy (PBK) undergoing DSAEK. Lamellar graft thickness was measured at the visual axis using anterior segment OCT, by the same person, at various time points after DSAEK. Eyes were divided into groups based on Day One postoperative endothelial lamella thickness: standard $(\leq 180 \mu \mathrm{m})$, medium-thick $(>180 \leq 250 \mu \mathrm{m})$ and thick $(>250 \mu \mathrm{m})$. Outcome measurements were graft survival rate, best spectacle-corrected visual acuity (BSCVA), endothelial cell density loss (ECD), and degree of astigmatism. Results in DSAEK eyes were compared to 20 PBK eyes which underwent PK.

Results: The median postoperative graft thickness of DSAEK eyes was $194.54 \pm 47.61 \mu$ m. There was no significant difference in age, sex, or preoperative BSCVA between DSAEK groups. The postoperative follow-up period was 18 months. Eyes with lamellar grafts of $\leq 180 \mu \mathrm{m}$ thickness showed better postoperative BSCVA and quicker recovery rates compared to the medium-thick and thick grafts $(P<0.001)$. Only eyes receiving $\leq 180 \mu m$ thickness lamellar grafts achieved equivalent BSCVA as PK eyes at month 18. Medium-thick grafts needed a longer period to obtain BSCVA scores similar to thinner grafts, while thick grafts never achieved the BSCVA of standard and PK grafts. All DSAEK eyes with lamella of thickness $\leq 180 \mu \mathrm{m}$, and only $50 \%$ of those with medium-thick lamella, had reached a BSCVA of $\geq 0.5$ at 6 months.
\end{abstract}

Conclusions: Lamella of thickness $\leq 180 \mu \mathrm{m}$ after DSAEK ensured a better and quicker visual recovery than thicker grafts.

Keywords: Endothelial keratoplasty; DSAEK; Lamellar thickness; Endothelial cell density loss

Abbreviations: DSAEK: Descemet Stripping Automated Endothelial Keratoplasty; PBK: Pseudophakic Bullous Keratoplasty; PK: Penetrating Keratoplasty; BSCVA: Best Spectacle Corrected Visual Acuity

\section{Introduction}

Penetrating keratoplasty $(\mathrm{PK})$ has been a gold standard of treatment for many corneal diseases for over a century. Many surgeons have posed a logical question; why should an entire cornea be transplanted, when in many corneal diseases only one corneal layer is affected and needs replacement? Even in 1950, Barraquer et al. [1] had proposed lamellar transplantation of the posterior cornea for endothelial diseases, but due to the technical difficulties it took time for this type of surgery to be widely accepted. Melles et al. [2-6] subsequently reported a novel surgical technique for posterior lamellar keratoplasty showing that the edematous cornea can be cleared if provided with a new functioning endothelial cell layer via a posterior corneal graft. The procedure was named "Descemet's Stripping with Endothelial Keratoplasty" or DSEK by Price et al. in USA. The lamellar cut was initially performed by manual lamellar dissection, but Gorovoy et al. [7] started performing lamellar cuts using a single pass of the microkeratome, naming the procedure DSAEK (Descemet's Stripping Automated Endothelial Keratoplasty). Today, almost $50 \%$ of corneal transplants in the USA and approximately $20 \%$ of those in Europe are lamellar surgeries - mostly DSAEK. Melles et al. [8-11] have investigated a further refinement of lamellar transplantation and initiated transplantation of the Descemet's membrane alone - "Descemet's membrane endothelial transplantation', or DMEK. The advantage of this procedure is significantly better visual acuity as compared with DSAEK; however the technique is technically more difficult and theoretically leads to higher endothelial cell density loss due to manipulation of the thin donor graft. Keeping in mind that DSAEK is surgically much safer and easier than DMEK, and that the thinner endothelial grafts bring quicker and better visual recovery, a so-called "ultra-thin DSAEK" with lamellar grafts of $50-80 \mu \mathrm{m}$ thickness made by double pass microkeratome was proposed as an optimal method [12]. This technique combines the advantages of DSAEK, where easier manipulation of the endothelial graft consequently decreases endothelial cell loss, and DMEK, where thin cut grafts bring better vision.

The goal of this study was to determine whether the endothelial graft thickness obtained by classic DSAEK, using a single pass microkeratome, affects visual acuity, visual recovery rate, graft survival rate and endothelial cell density loss. Therefore, we prospectively studied patients with PBK operated on by DSAEK and having different thicknesses of donor corneal lamella on the first postoperative day. The major outcomes were then compared over a follow-up period of 18 months.

\section{Subjects and Methods}

This study included a total of 40 eyes divided into two groups: patients that underwent endothelial keratoplasty - DSAEK $(n=20)$ and patients that underwent penetrating keratoplasty $(n=20)$ at The Eye

*Corresponding author: Iva Dekaris, University Eye Hospital "Svjetlost", Heinzelova 39, 10000 Zagreb, Croatia, Tel. 00385177756 56; Fax: 003851777 56 00; E-mail: iva.dekaris@inet.hr

Received November 03, 2011; Accepted January 27, 2012; Published February 01,2012

Citation: Dekaris I, Pauk M, Drača N, Pašalić A, Gabrić N (2012) Descemet Stripping Automated Endothelial Keratoplasty - Is a Thinner Donor Lamella the Better Choice? J Transplant Technol Res S2:004. doi:10.4172/2161-0991.S2-004

Copyright: ( 2012 Dekaris I, et al. This is an open-access article distributed under the terms of the Creative Commons Attribution License, which permits unrestricted use, distribution, and reproduction in any medium, provided the original author and source are credited. 
Hospital "Svjetlost". Preoperative indication for all patients was bullous keratopathy after cataract surgery - pseudophakic bullous keratopathy (PBK). There was no statistically significant difference in age, sex, or preoperative best spectacle corrected visual acuity (BSCVA) between groups. Only patients without any concomitant ocular diseases were included in the study; however in two eyes pupilar ectopia was present at the $12 \mathrm{o}^{\prime}$ clock position. $20 \mathrm{PBK}$ eyes that underwent DSAEK were prospectively followed-up over an 18 month period, and compared with the results of $20 \mathrm{PBK}$ eyes that had previously undergone penetrating keratoplasty $(\mathrm{PK})$ in the same setting by the same surgeon. Cutting of the donor grafts for DSAEK was performed via a single pass of a microkeratome (Moria 2, Moria S.A., France) by the operating surgeon. Prior to the microkeratome cut, the thickness of the donor cornea was measured, and either a 300 or $350 \mu \mathrm{m}$ head was used depending on the central corneal thickness of the donor graft. For values up to $600 \mu \mathrm{m}$, the $300 \mu \mathrm{m}$ head was used, and for thicker donor corneas a $350 \mu \mathrm{m}$ head was used. Donor corneal tissues were obtained from certificated eye banks in Europe, and stored in organ-culture prior to lamellar cut. They were carefully selected, under central corneal density criteria, for possessing greater than 2500 cells $/ \mathrm{mm}^{2}$. DSAEK patients $(\mathrm{n}=20)$ were then divided into three subgroups according to the postoperative thickness of the lamellar graft on Day One postoperative: the standard thickness lamella group was $\leq 180 \mu \mathrm{m}(\mathrm{n}=10)$, the medium-thick group was $180-250 \mu \mathrm{m}(\mathrm{n}=8)$, and finally lamella $>250 \mu \mathrm{m}$ were classified as the thick group $(n=2)$. The thickness of a lamellar graft was measured only after surgery, and performed manually by the same person at the visual axis of each graft. Non-contact optical coherence tomography customized for the anterior segment (Zeiss Visante ${ }^{\mathrm{Tx}}$ ASOCT, Germany) was used to measure the thickness of donor grafts on the first day, and then one, two, three, six, nine, twelve, and eighteen months postoperatively. Best corrected visual acuity according to the standard Snellen chart, postoperative refractive error by subjective and objective refraction, and topography, were also measured. Influence of the postoperative thickness of the lamellar graft on visual recovery was evaluated, and the data were statistically analyzed by Student t-test. $\mathrm{P}<0.005$ was considered statistically significant. DSAEK outcomes were additionally compared to $\mathrm{PK}$ results regarding graft survival rate, postoperative astigmatism and visual acuity. The mean followup period was 18 months (range 15-19 months) for DSAEK, and 24 months (range 16-28 months) for PK group. Data were compared regarding visual outcome, endothelial cell count, complications, and survival rate.

\section{Results}

The median postoperative graft thickness of eyes that underwent DSAEK was $194.54 \pm 47.61 \mu \mathrm{m}$. The DSAEK group was divided into three subgroups according to the postoperative thickness of grafted lamella: 1) the standard lamella group was $\leq 180 \mu \mathrm{m}$, with an average thickness of $152 \pm 19.23 \mu \mathrm{m}, 2)$ the medium-thick group was 180-250 $\mu \mathrm{m}$, with an average thickness of $215 \pm 23.80 \mu \mathrm{m}$ and 3) thick lamellas were defined as $>250$ with an average thickness of $260 \pm 14.00 \mu \mathrm{m}$.

DSAEK grafts of standard thickness $(\leq 180 \mu \mathrm{m})$ showed significantly better postoperative BSCVA both in terms of eventual maximum value and in recovery rate as compared to medium-thick and thick grafts $(\mathrm{P} \leq 0.05)$. Only eyes with grafts $\leq 180 \mu \mathrm{m}$ achieved BSCVA better or equal to $\mathrm{PK}$ eyes 18 months after surgery. Medium-thick grafts required longer rehabilitation times (up to six months) to obtain BSCVA values similar to standard grafts, while thick grafts never reached the BSCVA of standard DSAEK and PK grafts (Figure 1). All DSAEK eyes of standard thickness had BSCVA $\geq 0.5$ at six months after surgery. In contrast, only $50 \%$ of the medium-thick and none of the thick DSAEK grafts reached a BCVA $\geq 0.5$ by six months after surgery; with very modest improvements in visual acuity up to 18 months. Anterior segment OCT pictures representative of each DSAEK group are shown in Figures 2, 3 and 4. Eyes with lamella of $\leq 180 \mu \mathrm{m}$ at Day One postop had even thinner lamella at one month after surgery (Figure 2), while medium-thick and thick lamella slowly became thinner and had attained similar thickness as standard grafts at three and six months after surgery, respectively (Figure 3 and 4). The slit lamp appearances of standard and medium-thick lamella in a patient eye at Day One postoperative and at three months after surgery are shown in Figure $5 \mathrm{a}$ and $5 \mathrm{~b}$. The difference in corneal transparency is visible at Day One postoperative but not at three months after surgery, by which time the BSCVA values between these grafts had become very similar. The overall mean spherical equivalent in DSAEK eyes showed a hyperopic shift of $+1.5 \mathrm{D} \pm 1.0 \mathrm{D}$, while in PK eyes it was $-2.75 \pm 2.0 \mathrm{Dpt}$. Thicker donor buttons and meniscus shaped donor buttons produced more hyperopic shift. Refractive astigmatism was significantly lower in the DSAEK group $(1 \pm 0.75 \mathrm{D})$ compared to the PK group $(3.4 \pm 1.5 \mathrm{D})$, $(\mathrm{P} \leq 0.05)$ (Figure 6).

As expected, DSAEK grafts had greater ECD loss than PK grafts within the first month after the procedure. However, 18 months after surgery there was no significant difference in endothelial cell density loss between the PK (39.9\%) and DSAEK grafts (average: $40.1 \%$ ) or between DSAEK subgroups (37.9\%, 38.7\% and $43.7 \%$ for groups 1,2 and 3, respectively) (Figure 7). Complications in the DSAEK group included: one irregular cut - namely, a cut in which the difference between central and peripheral lamellar thickness was greater than 200 $\mu \mathrm{m}$ and one IOP increase at 6 hours after surgery. The IOP increase was resolved immediately by air bubble release. None of the DSAEK grafts detached; the air bubble was left in the eye for the first two postoperative days, apart from the patient with a postoperative IOP increase. The most frequent postoperative complications in the $\mathrm{PK}$ group were high astigmatism, followed by increased intraocular pressure (two patients) and persistent epithelial defect (three patients).

\section{Discussion}

Endothelial keratoplasty(EK) is the selective replacement of diseased endothelium with a healthy donor endothelium. More and more surgeons are performing EK because it is a safer surgery as compared to

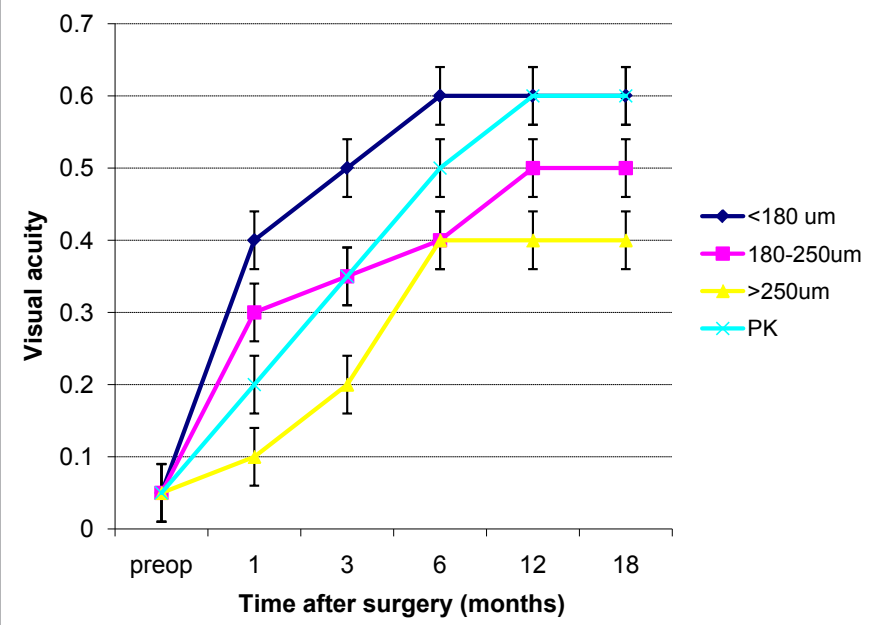

Figure 1: Best corrected visual acuity in eyes operated on due to pseudophakic bullous keratopathy by either: 1. Descemet stripping automated endothelial keratoplasty (DSAEK) - with different thicknesses of donor endothelial lamella $(\leq 180 \mu \mathrm{m}, 180-250 \mu \mathrm{m},>250 \mu \mathrm{m}$ ) $)$ or 2 . Penetrating keratoplasty (PK). 
Citation: Dekaris I, Pauk M, Drača N, Pašalić A, Gabrić N (2012) Descemet Stripping Automated Endothelial Keratoplasty - Is a Thinner Donor Lamella the Better Choice? J Transplant Technol Res S2:004. doi:10.4172/2161-0991.S2-004

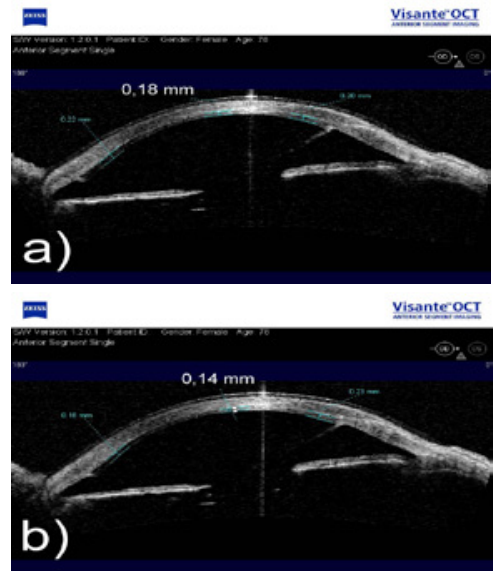

Figure 2: Standard lamella of DSAEK graft on: a) Day One postoperative; b) one month after surgery.
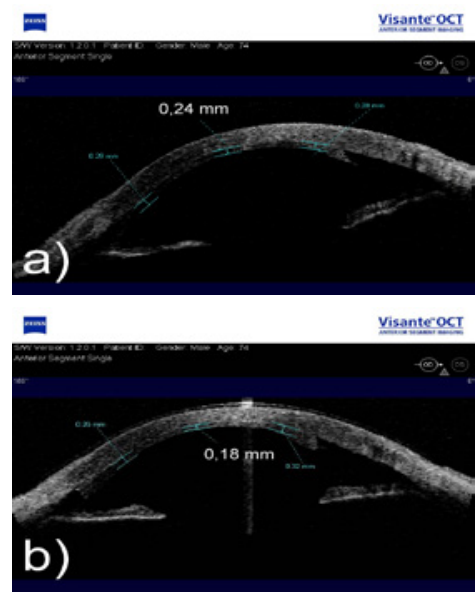

Figure 3: Medium-thick lamella of DSAEK graft on: a) Day One postoperative; b) three months after surgery.
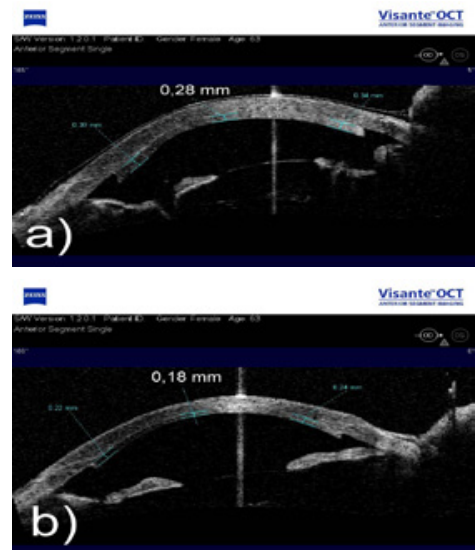

Figure 4: Thick lamella of DSAEK graft at: a) Day One postoperative; b) six months after surgery.

PK. The smaller incision made for DSAEK results in a normal tectonic strength in the eye, with higher resistance to traumatic rupture for the rest of the patient's life. Moreover, DSAEK patients are more satisfied with their UCVA compared to those who undergo PK, especially regarding visual recovery rate [5,13-17]. Stable visual acuity does not occur for at least six months to one year after a conventional PK, and sometimes requires even longer. With DSAEK there is a faster recovery, and most patients have usable vision within six weeks after operation; some have excellent vision after just one week [13-17]. The experience with DMEK grafts, where only the Descemet's membrane is grafted, shows that very thin grafts provide significantly better vision quickly after surgery; and similar results are reported for so called "ultra-thin" DSAEK $[11,12]$. Having this in mind, we have prospectively followedup patients with bullous keratopathy undergoing conventional DSAEK in order to determine whether the postoperative thickness of donor lamella influences the speed and degree of visual recovery. The factors that can affect the thickness of a donor lamella include the thickness of a donor cornea, donor corneal curvature, backpressure in the artificial anterior chamber, and the translational speed of the microkeratome [16]. Because of these influencing factors, we have always measured the thickness of the donor cornea prior to cut, and selected the microkeratome head accordingly, but other factors could not be fully controlled. Patients were divided into 3 DSAEK subgroups according to the Day 1 lamellar thickness measured by OCT at the visual axis. As shown by our results, it seems that a donor lamella of $\leq 180 \mu \mathrm{m}$ ensures quicker visual recovery and better overall vision in the long run as compared with thicker lamellas (of over $180 \mu \mathrm{m}$ ). All DSAEK eyes with a donor lamella of $\leq 180 \mu \mathrm{m}$ had BSCVA $\geq 0.5$ at six months after surgery, similar to other published results [17-20]. In a study published by Neff et al. [19], the median postoperative thickness of all eyes was $131 \mu \mathrm{m}$, and the authors compared the visual acuity of thin lamellas averaging $109 \mu \mathrm{m}$ with thick lamellas averaging $162 \mu \mathrm{m}$, concluding that thinner lamellas bring statistically significant improvement in BSCVA. Although this conclusion is similar to ours, these results are not directly comparable since the median lamellar thicknesses are different between two studies. In our study, only $50 \%$ of medium-thick DSAEK grafts reached a BCVA of $\geq 0.5$ at 6 months after surgery, supporting our hypothesis that thicker grafts can influence final visual acuity outcomes after DSAEK. Finally, although the number of cases was very small $(n=2)$, we have shown that beyond a critical point of donor
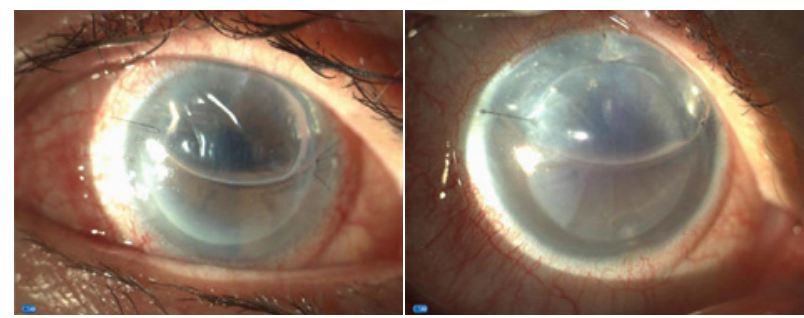

Figure 5a: Patient with a standard (left) and medium-thick (right) endothelial lamella on the first postoperative day after DSAEK for pseudophakic bullous keratopathy (visible difference in corneal transparency).
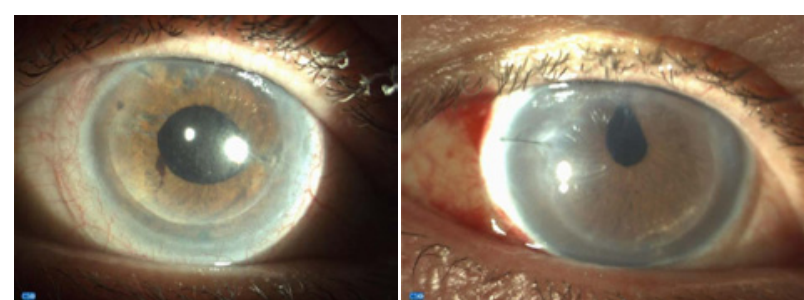

Figure 5b: Patient with a standard (left) and medium-thick (right) endothelial lamella at three months after DSAEK for pseudophakic bullous keratopathy (there is no visible difference in corneal transparency). 


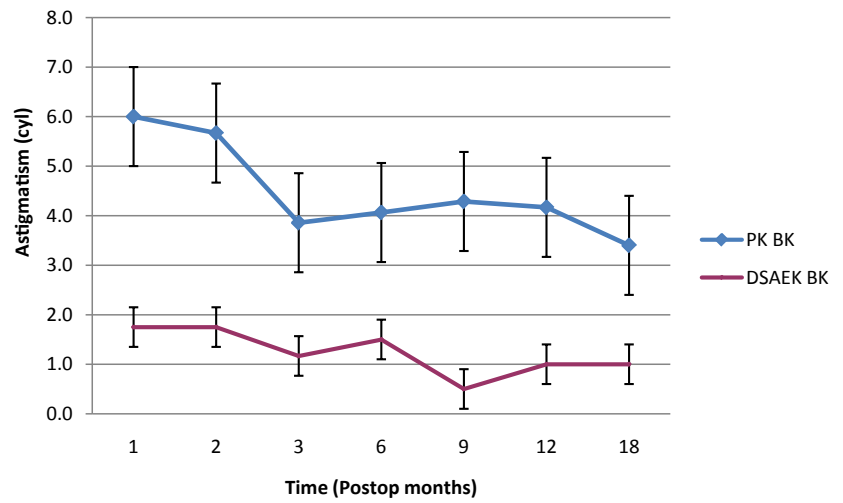

Figure 6: Mean refractive astigmatisms after DSAEK and penetrating keratoplasty (PK) for pseudophakic bullous keratopathy.

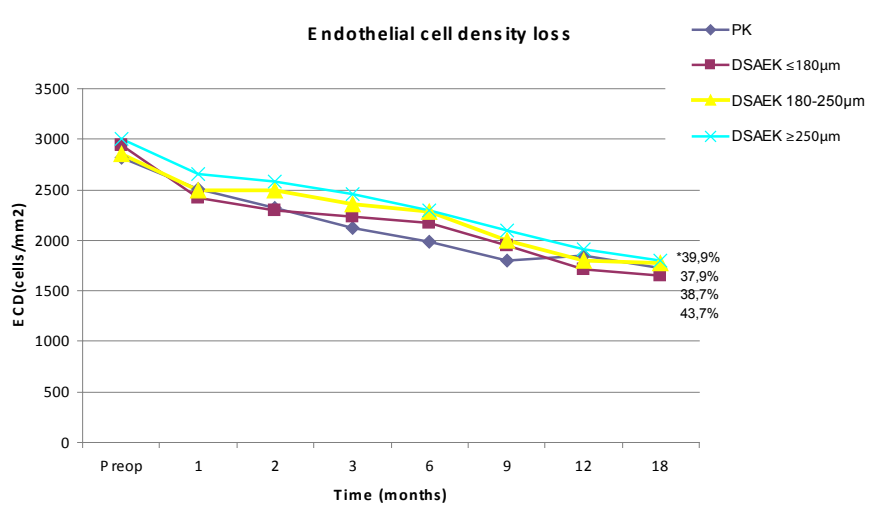

*Endothelial cell loss percentage for each group in order of appearance in the chart.

Figure 7: Postoperative endothelial cell density loss in patients operated on due to pseudophakic bullous keratopathy, either by DSAEK or PK.

lamella thickness $(>250 \mu \mathrm{m})$, BSCVA will not equalize with thinner lamellar grafts or conventional PK over 18 postoperative months. The difference in BSCVA between DSAEK subgroups after six months of follow-up cannot be explained by the early postoperative thickness of donor lamellas, since thicker lamellas slowly but consistently became thinner. Namely, at 6 months the AS-OCT-measured thickness of lamellar grafts at visual axis was not significantly different between standard, medium-thick and thick grafts. Also, when looking at the corneal transparency via slit-lamp, one could hardly see the difference between standard, medium-thick, and thick lamellar grafts after six months or a year; but the difference in BSCVA was still present. One can presume that donor lamellar grafts which are thicker in the early postoperative period will produce more interface problems compared to thinner grafts, resulting in lower BSCVA in thicker grafts, even after six months when they have regain their standard thickness. However, before making any final conclusions, one must keep in mind that we have observed only small number of grafts (especially thick ones), so the data should be confirmed in a larger number of cases. Moreover, there is a published study on 37 eyes in which the authors have found no direct effect of lenticule thickness on 6-month postoperative visual acuity, contrary to our study and with a similar average donor lamella thickness of $175 \mu \mathrm{m}$ [21].

Another important factor influencing the quality of vision after transplantation is the degree of astigmatism, which is highly unpredictable in $\mathrm{PK}$ and commonly 3 to $5 \mathrm{D}$ of mean refractive cylinder, while after DSAEK patients can typically expect up to $1.5 \mathrm{D}$ $[7,17,20]$. According to our results, the mean refractive cylinder after DSAEK was $1 \mathrm{D}$, as compared to PK where it was $3.4 \mathrm{D}$, which is similar with other published data $[17,20]$. It is well known that DSAEK causes a mild hyperopic shift in the mean spherical equivalent. This can be explained by two characteristics of the donor graft: the central corneal thickness and the thickness gradient from center to periphery. Thicker donor buttons and meniscus shaped donor buttons tend to produce greater hyperopic shift, while thinner donor buttons can produce even myopic shift. In our group of patients, all DSAEK eyes showed hyperopic shifts without any significant difference between eyes with standard, medium-thick and thick lamellar grafts.

As previously mentioned, endothelial keratoplasty has several advantages over PK. However, the major concern of many surgeons has been greater endothelial cell density loss during EK, which has been reported to be between $24 \%$ and $50 \%$ at six months to one year after DSAEK; this is indeed higher than the early cell loss reported in most PK series [20,21]. The early cell loss with DSAEK is not surprising, because it entails more donor tissue manipulation than PK. However, studies with longer follow-up have shown that after 3-4 years ECD cell loss was similar in DSAEK and in PK [22]. In our study, we have compared ECD loss between standard, medium-thick, and thick DSAEK grafts and PK grafts. We have observed higher ECD loss at one month after surgery in all DSAEK eyes, most probably due to more tissue manipulation during surgery, but the ECD loss equalized during a longer follow-up period of 18 months with the loss after PK. We have seen no difference in ECD loss between standard, medium-thick, and thick donor lamellas. Therefore, the observed difference of BSCVA among DSAEK eyes with different thicknesses of donor lamella cannot be attributed to the state of the endothelium in each subgroup.

According to our results on a relatively small number of eyes, thinner lamellar endothelial grafts bring better vision, sooner after surgery, as compared to thick lamellar grafts or PK. Our study also confirmed that DSAEK was superior to PK in the endpoint measures of corneal astigmatism and visual acuity recovery rate; and that it is a safe procedure since only 2 complications occurred without any consequences for the final visual outcome. The most frequent DSAEK complication, lamellar detachment, did not occur, probably because we kept the air-bubble in the anterior chamber longer than usual [23]. Finally, the endothelial cell density loss in all DSAEK eyes, regardless of the early postoperative lamellar thickness, was equal to that in PK eyes in a follow-up period longer than 12 months.

\section{References}

1. Barraquer JI (1951) Queratoplastia: Problemas que plantea la fijacion de injerto. 16 th Concilium Ophtalmologicum Acta. London. British Medical Assosiation 999-1004.

2. Melles GR, Eggink FA, Lander F, Pels E, Rietveld FJ, et al. (1998) A surgica technique for posterior lamellar keratoplaty.Cornea 17: 618-626.

3. Melles GR, Lander F, Beekhuis WH, Remeijer L, Binder PS (1999) Posterior lamellar keratoplasty for a case of pseudophakic bullous keratopathy. Am J Ophthalmol 127: 340-341.

4. Melles GR, Lander F, Nieuwendaal C (2002) Suturless, posterior lamellar keratoplasty: a case report of a modified technique. Cornea 21: 325-327.

5. Melles GR, Lander F, van Dooren BT, Pels E, Beekhius WH (2000) Preliminary clinical results of posterior lamellar keratoplasty through a sclerocorneal pocket incision. Ophthalmology 107: 1850-1856.

6. Melles GR, Kamminga N (2003) Techniques for posterior lamellar keratoplasty through a scleral incision. Ophthalmologe 100: 689-695.

7. Gorovoy MS (2006) Descemet stripping automated endothelial keratoplasty Cornea 26: 886-889. 
Citation: Dekaris I, Pauk M, Drača N, Pašalić A, Gabrić N (2012) Descemet Stripping Automated Endothelial Keratoplasty - Is a Thinner Donor Lamella the Better Choice? J Transplant Technol Res S2:004. doi:10.4172/2161-0991.S2-004

8. Melles GR, Lander F, Rietveld FJ (2002) Transplantation of Descemet's membrane carrying viable endothelium through a small scleral incision. Cornea 21: $415-418$

9. Melles GR (2006) Posterior lamellar keratoplasty: DLEK to DSEK to DMEK Cornea 25: 869-881.

10. Melles GR, Ong TS, Ververs B, van der Wees J (2006) Descemet membrane endothelial keratoplasty (DMEK). Cornea 25: 987-990.

11. Melles GR, Ong TS, Ververs B, van der Wees J (2008) Preliminary clinica results of Descemet membrane endothelial keratoplasty. Am J Ophthalmo 145: 222-227.

12. Sikder S, Nordgren RN, Neravetla SR, Moshirfar M (2011) Ultra-thin donor tissue preparation for endothelial keratoplasty with a double-pass microkeratome. Am J Ophthalmol 152: 202-208

13. Terry MA (2005) “Endothelial Replacement Surgery" In: Krachmer J, Mannis M Holland E eds. Cornea: surgery of the cornea and conjunctiva. St. Louis: 2nd edition, Elsevier Mosby 140: 1707-1718.

14. Terry MA, Ousley PJ (2005) Deep lamellar endothelial keratoplasty (DLEK) Visual acuity, astigmatism, and endothelial survival in a large prospective series. Ophthalmology 112: 1541-1549.

15. Price FW, Price MO (2005) Descemet's stripping with endothelial keratoplasty in 50 eyes: A refractive neutral corneal transplant. J Refract Surgery 21: 339 345
16. Terry MA (2006) Endothelial Keratoplasty (EK) History, Current State and Future Directions. Cornea 25: 873-878.

17. Koenig SB, Covert DJ (2007) Early results of small - incision Descemet's stripping and automated endothelial keratoplasty. Ophtalmology 114: 221-226.

18. Chen ES, Terry MA, Shamie N, Hoar KL, Friend DJ (2008) Descemet-stripping automated endothelial keratoplasty: six-month results in a prospective study of 100 eyes. Cornea 27: 514-520.

19. Neff KD, Biber JM, Holland EJ (2011) Comparison of central corneal graft thickness to visual acuity outcomes in endothelial keratoplasty. Cornea 30 : 388-391.

20. Koenig SB, Covert DJ, Dupps WJ Jr, Meisler DM (2007) Visual acuity, refractive error and endothelial cell density six months after Descemet stripping automated endothelial keratoplasty (DSAEK). Cornea 26: 670-676.

21. Van Cleynenbreugel H, Remeijer L, Hillenaar T (2011) Descemet stripping automated endothelial keratoplasty: effect of intraoperative lenticule thickness on visual outcome and endothelial cell density. Cornea 30: 1195-1200.

22. Lass JH, Gal RL, Dontchev M, Beck RW, Kollman C, et al. (2008) Donor age and endothelial cell loss 5 years after successful corneal transplantation. Specular microscopy ancillary study results. Ophthalmology 115: 627-632.

23. Price MO, Price FW Jr (2006) Descemet's stripping with endothelial keratoplasty in 200 eyes: early challenges and techniques to enhance donor adherence. $J$ Cataract Refract Surg 32: 411-418.

This article was originally published in a special issue, Endothelia Keratoplasty handled by Editor(s). Dr. Jodhbir S Mehta, Singapore Eye Research Institute, (SERI), Singapore; Dr. Saijad Ahmad, Newcastle University, United Kingdom 